\title{
The Role of High-Frequency Oscillatory Activity in Reward Processing and Learning
}

\author{
Josep Marco-Pallarés ${ }^{1,2}$, Thomas F. Münte ${ }^{3,4}$, Antoni Rodríguez-Fornells ${ }^{1,2,5}$ \\ ${ }^{1}$ Department of Basic Psychology, University of Barcelona, 08035 Barcelona, Spain \\ ${ }^{2}$ Cognition and Brain Plasticity Unit. Bellvitge Research Biomedical Institute (IDIBELL), \\ Hospitalet de Llobregat, Barcelona, Spain \\ ${ }^{3}$ Department of Neurology, University of Lübeck, Lübeck, Germany \\ ${ }^{4}$ Institute of Psychology II, University of Lübeck, Lübeck, Germany \\ ${ }^{5}$ Institució Catalana de Recerca i Estudis Avançats (ICREA), Barcelona, Spain
}

\section{Corresponding author}

Josep Marco-Pallares

Facultat de Psicologia Campus de Mundet

Edifici Ponent, Universitat de Barcelona

Passeig de la Vall d'Hebron 171

08035 Barcelona

e-mail: josepmarco@gmail.com

Phone: (+34) 933125156 


\begin{abstract}
Oscillatory activity has been proposed as a key mechanism in the integration of brain activity of distant structures. Particularly, high frequency brain oscillatory activity in the beta and gamma range has received increasing interest in the domains of attention and memory. In addition, a number of recent studies have revealed an increase of betagamma activity $(20-35 \mathrm{~Hz})$ after unexpected or relevant positive reward outcomes. In the present manuscript we review the literature on this phenomenon and we propose that this activity is a brain signature elicited by unexpected positive outcomes in order to transmit a fast motivational value signal to the reward network. In addition, we hypothesize that beta-gamma oscillatory activity indexes the interaction between attentional and emotional systems, and that it directly reflects the appearance of unexpected positive rewards in learning-related contexts.
\end{abstract}

KEYWORDS: oscillatory activity, reward, novelty, beta, gamma 


\section{Introduction}

Oscillatory activity is a basic brain mechanism that allows communication between distant brain areas (Buzsaki and Draguhn, 2004). In the last decade, oscillatory activity in the beta (12 to $30 \mathrm{~Hz})$ and gamma (>30 Hz) frequency bands of mammals has been found to be crucial for information processing in the central nervous system and has been related to a variety of motor and cognitive functions including perception, motor control, sensorimotor integration, attention, inhibition, memory and higher level cognition (Chen et al., 2004; Jensen et al., 2007; Llinás et al., 2005; Ribary, 2005; Schnitzler and Gross, 2005; Uhlhaas and Singer, 2006; Wang, 2010). Recently, human electrophysiological studies have described an increase of high beta-low gamma oscillatory activity after reward delivery in gambling and learning tasks (Cohen and Ranganath, 2007; Doñamayor et al., 2012, 2011; Marco-Pallares et al., 2008; MarcoPallarés et al., 2009).

In the following sections we will review the recent literature on reward processing in humans to propose the hypothesis that beta-gamma oscillatory activity is mediating the interaction between brain systems involved in attention and reward processing, and that it directly reflects the appearance of unexpected positive rewards in learning related contexts. Thus, beta-gamma oscillatory activity will appear whenever a feedback stimulus conveys new information about unexpected positive and significant rewards. This brain oscillatory signature might signal the need to store new information in memory for guiding future actions and, therefore, might represent a key mechanism for orchestrating the cross-talk between learning processes, involving the medial temporal lobe, the ventral striatum, medial prefrontal regions and the midbrain dopaminergic system.

\section{Functional role of Beta-Gamma activity}

Studies on reward (Knutson et al., 2005; O'Doherty et al., 2001) or positive feedback processing (Camara et al., 2010, 2009; Nieuwenhuis et al., 2005) have revealed an extended network including the ventral striatum, amygdala, insula, ventromedial prefrontal cortex and anterior cingulate cortex among others. However, the mechanisms that allow the integration of information of the different nodes of this network are largely unknown. Using electroencephalography (EEG) to record the brain electrical 
activity of large neuronal groups, two independent studies using different experimental paradigms reported an increase of oscillatory activity when participants received a signal indicating a monetary gain (positive feedback, Cohen et al., 2007; Marco-Pallares et al., 2008; Figure 1B). This increase occurred 200 to $400 \mathrm{~ms}$ after the feedback informing about the positive outcome, and was produced at frequencies ranging from 20 to $35 \mathrm{~Hz}$ which are traditionally considered to represent high beta-low gamma frequency ranges ( $\beta \gamma$ activity from now on). Marco-Pallares et al., (2008) used a gambling task in which the subjects had to choose between a larger and a smaller number that corresponded to the win or loss of the equivalent sum in Euro cent $(p=0.5$ for wins and losses, Figure 1A). In this study the win-related $\beta \gamma$ power was more pronounced for larger rewards than smaller rewards, suggesting that this activity could be related to the magnitude of the outcome.

Similarly, Cohen and colleagues (2007) also found a greater $\beta \gamma$ power after positive feedback. Here, a reversal learning task was used, in which participants had to choose between two squares located at left or right positions. After the selection, a visual feedback indicated whether the participant received a reward (10 cents) or a punishment (-10 cents). During a block (80 to 150 trials) each of the sides was associated to a probability to win. When a new block started, and without informing to the participant, the probabilities changed. The three possible probabilities for left and right locations were, for different blocks, $0,75-0,25 ; 0,5-0,5$ and 0,25-075 respectively. Interestingly, $\beta \gamma$ oscillatory activity was more pronounced for unexpected rewards $(\mathrm{p}($ win $)=0.25)$ than for expected rewards ( $\mathrm{p}($ win $)=0.75)$. Previously, Keil and collegues (2001) had also described a similar activity using a paradigm in which participants had to press a button three seconds after a cue and in order to receive a monetary reward. The amount of money depended on how temporally precise the response was. Upon the button press, which was immediately followed by the presentation of feedback indicating the amount of reward, left frontal electrodes showed an increase of power in the $20-30 \mathrm{~Hz}$ frequency range, 350 to $500 \mathrm{~ms}$ after the button press. This activation was not present when the reward was delivered randomly or when participants just pressed the button, suggesting that the effect was not related to motor activity. Interestingly, in a recent magnetoencephalography (MEG) gambling study, Doñamayor et al., (2011) also found an increase in $\beta \gamma$ power (with peaks at 29 and $34 \mathrm{~Hz}$ ), 200 to $500 \mathrm{~ms}$ after reward delivery (Figure 1C, Doñamayor et al., 2012, 2011). 
These initial studies clearly pointed out the involvement of reward-related processes in the generation or modulation of the $\beta \gamma$ component (Marco-Pallarés et al., 2008). This proposal was further supported by another study showing that the $\beta \gamma$ increase after monetary gains was related to the Catechol-O-methyltransferase enzyme (COMT) Val158Met polymorphism (Marco-Pallarés et al., 2009). COMT plays a key role in the degradation of dopamine in the prefrontal cortex. Bilder et al. (2004) proposed an inverse relationship between tonic dopaminergic activity in the prefrontal cortex and phasic activity in striatal areas. Therefore, carriers of the Met allele, which has been associated to low enzyme activity (that is, reduced dopamine degradation in the prefrontal cortex), would show increased tonic activity in the prefrontal cortex which would lead to decreased dopaminergic striatal phasic activity. In contrast, participants homozygous for the Val allele would show decreased prefrontal tonic activity and increased striatal phasic activity. Indeed, participants homozygous for the Val allele showed enhanced $\beta \gamma$ oscillatory activity after gains compared to MetMet carriers, supporting the tonic-phasic hypothesis. Further, Padrão et al. (2013) also showed that individuals with high trait anhedonia presented a smaller $\beta \gamma$ response after rewards than high hedonic participants. Anhedonia is the reduction of the ability to experience pleasure (Meehl, 1975) and has been related to dysfunctions in the reward system, especially in the Nucleus Accumbens (Ruso and Nestler, 2013). In addition, following similar rationale, a recent study found a positive correlation between this activity and the trait of sensation seeking (Leicht et al., 2013). Both human studies and animal models have shown that high sensation seekers present and increased mesolimbic dopaminergic activity (Blanchard et al. 2009), especially in the Nucleus Accumbens. These results support the involvement of the reward network and its dopaminergic subcortical components in the generation of $\beta \gamma$ oscillatory activity.

However, while initial studies had shown that $\beta \gamma$ oscillatory activity was elicited by positive reward outcomes, it became subsequently clear that not all positive reward conditions elicit this activity. In HajiHosseini et al., (2012) we investigated its relationship to the difference between expected and obtained rewards and punishments (prediction error) by employing a gambling task in which a cue indicated the probability and magnitude of upcoming outcomes (monetary gains or losses, Figure 2A). The goal of the experiment was to determine whether $\beta \gamma$ activity was modulated by the probability, magnitude or the expected value of the monetary outcome. A $\beta \gamma$ oscillatory 
activity increase was observed only after unexpected gains, that is, those gains with low probability (Figure 2A). In addition, $\beta \gamma$ activity was not related to the expected value, prediction error or to the magnitude of the reward. This result also agrees with a previous finding of an increase of $\beta \gamma$ power after improbable rewards (Cohen et al. 2007). In addition, Cunillera et al. (2012) analyzed feedback processing in a modified version of the Wisconsin Card Sorting Task that required the selection of one of four cards according to a certain rule (color, shape, number, Figure 2B). After each selection the participant is informed whether the selection has been correct or incorrect. However, at a certain moment, and without informing the participant, the rule changes and the participant has to infer the new rule by trial and error. The study revealed that the $\beta \gamma$ increase appeared only following the first positive feedback after a correct rule change. In other words, $\beta \gamma$ activity appeared after the most informative positive feedback, the one that indicated that the correct rule had been selected. Another relevant study analyzed the effect of contextual novelty in reward processing (Bunzeck et al., 2011). Participants had to learn the relationship of different fractal images to the probability of winning money and subsequently performed a recognition task that required them to determine whether a scene was indoor or outdoor. Some of these scenes were familiar (had been presented in a previous phase of the experiment) and others were novel. After the decision, the fractal image appeared indicating the probability of subsequent monetary reward. $\beta \gamma$ activity was enhanced for fractal images indicating high reward probability (Figure 2C) and furthermore this activity was modulated by the familiarity of the context, being higher for the novel condition. According to the authors, these results supported the idea that contextual novelty increased the activation of rewardpredicting cues. Complementary results were found by Kawasaki and Yamaguchi (2013) using a monetary-incentive, delayed-response task in which participants had to remember the position of colored disks. In the delay period when participants were informed about the amount of money they could win, beta activity (around $24 \mathrm{~Hz}$ ) was higher for high reward trials compared to low reward trials.

Overall, these results suggest that $\beta \gamma$ modulations selectively track salient and novel positive events in the environment and thus reflect a general monitoring mechanism for unexpected positive events. Indeed, gamma oscillatory activity has been related to attention (Herrmann and Knight, 2001) and novelty detection (Lapray et al., 2009). Importantly, $\beta \gamma$ activity in reward tasks are sensitive to the valence of events, as 
better than expected outcomes elicit such activity while worse than expected outcomes do not. Thus, $\beta \gamma$ activity behaves unlike other oscillatory components such as theta band power increase associated with unsigned prediction error (that is, discrepancies between expected and real outcomes; Cavanagh et al., 2012; Mas-Herrero and Marco-Pallarés, 2014). Hence, the properties of $\beta \gamma$ activity do not fit with computational accounts proposing that some brain signals generated in the medial prefrontal cortex regions (such as Anterior Cingulate Cortex) encode the surprise of an outcome independently from its valence (Alexander and Brown, 2011). In fact, $\beta \gamma$ activity echoes the behavior of neurons in the Ventral Tegmental Area (VTA) of conscious monkeys which increase their firing rate after improbable (Fiorillo et al., 2003) or larger than expected rewards (Tobler et al., 2005). In contrast, unexpected or improbable punishments produced a decrease in the phasic activity of VTA dopaminergic neurons (Fiorillo et al., 2003; Schultz et al., 1993; but see Matsumoto and Hikosaka, 2009, for neurons in the SN/VTA firing at both positive and negative outcomes).

\section{Beta-gamma activity in the interplay between attention and memory in reward processing}

Considering the previously reviewed evidence, we propose that the increase in $\beta \gamma$ oscillatory activity represents a brain signature elicited by unexpected positive outcomes and in charge of transmitting a fast motivational value signal to downstream brain structures (Figure 3). This type of information might be crucial in determining changes in the environment and in adapting behavior to new contingencies. In addition, it requires the interplay of attention mechanisms (novelty processing), reward processing and memory (storing action plans and learning for future episodes). This oscillatory activity might be a key component in mediating cross-talk between these processes.

Regarding the relationship between attention and reward processing, it is well known the important role of expectancy and reward anticipation in selecting among different response options as well as the role of surprise and novelty at reward occurrence (Mellers and McGraw, 2001; Mellers et al., 1998). Reward processing and attention continuously interact, in particular when expecting improbable but highly 
desired outcomes (Fuentemilla et al., 2013). Orienting responses are triggered to novel and unexpected events (Sokolov, 1963; Donchin, 1981), especially those events that are very relevant to on-going goals. Disconfirmations of important expected events enhance attentional processing (Olson et al., 1996), increase physiological arousal (Berlyne, 1960), trigger dopaminergic VTA activity in the midbrain reward pathways (Schultz et al., 1997) and amplify emotional reactivity to those events (Schachter and Singer, 1962; Zillmann, 1978; Ortony et al., 1988). Importantly, this emotional reactivity reinforces attention (Anderson, 2005; Pessoa et al., 2002) in order to increase the likelihood of remembering for future occasions (Ohman et al., 2001). Besides, rewarding signals also impact directly into the alerting system, triggering attentional processes and energizing the organisms in order to correctly execute or avoid certain actions (Berridge and Robinson, 1998; Pessiglione et al., 2007). In this sense, increasing the amount of attention and alertness of the system might be important to capture more easily unexpected signals in the environment that could be of emotional relevance for the organism, especially under new, unexpected or uncertain situations (Fiorillo et al., 2003). As stated above, gamma band oscillatory activity has been associated with increased attentional processing which might reinforce the neural representations associated with attended (unexpected) stimuli and facilitate its processing and store through the communication of distant neural groups (Buschman and Miller, 2009; Gregoriou et al., 2009; Womelsdorf and Fries, 2007).

Complementarily to our proposal, Cohen et al., (2011) proposed that plastic changes associated with learning from feedback could be associated with the synchronization of distant areas through oscillatory activity. Accordingly, synaptic changes associated with learning from positive feedback would be reflected in changes in $\beta \gamma$ synchronization, while synaptic plasticity associated with learning from negative events would lead to changes in the theta range (4-8 Hz). These two different oscillatory mechanisms would also engage different neural systems: while $\beta \gamma$ activity would be generated in the ventromedial prefrontal cortex, the theta oscillatory activity associated with negative feedback likely emanates from the dorsal Anterior Cingulate Cortex. Similarly, but using a theoretical connectionism model, Phaf and Rotteveel, )2012) proposed that positive affect was conveyed by high frequency oscillatory activity while negative affect would correspond to lower frequencies in the theta range. Finally, van de Vijver et al.(2011) stressed that beta activity also fulfilled the characteristics of a 
learning signal. In line with the idea coming from animal work that beta oscillations signal the tendency to maintain "the status quo" (Engel and Fries, 2010), the beta increase after positive feedback was interpreted as a signal to strengthen the current response set rather than other options, thereby influencing future behavior.

Crucial to our hypothesis, Axmacher et al. (2010) found a gamma increase in the hippocampus and ventral striatum (Nucleus accumbens, NAcc) to unexpected relevant items that had to be coded in long term memory. Following the proposal of Lisman and Grace (2005) that novelty detection is coded in the hippocampus, which in turn sends the information to the VTA via the NAcc and ventral pallidum, Axmacher et al. (2010) speculated that the hippocampus - NAcc - VTA dopaminergic loop might support the detection and storage of unexpected events using gamma oscillatory activity. The activation of this loop would be modulated by the VTA via the release of dopamine in the NAcc (Legault and Wise, 2001) and this information would be crucial in the establishment of new predictions about the environment based on the coupling of several regions using this high-frequency oscillatory activity. We propose that this loop might process unexpected events differently based on their emotional valence. We further speculate that $\beta \gamma$ activity associated with reward processing is modulated by the ventral striatum or VTA dopaminergic activity. Support for this speculation can be derived from the effect of dopamine polymorphisms on $\beta \gamma$ oscillations (Marco-Pallarés et al., 2009) and from the relationship between dopamine activity and changes from low to high gamma oscillatory activity (Brown et al., 2001; Lalo et al., 2008).

These ideas are also corroborated by intracranial animal data. An increase of activity in these frequency bands after reward delivery has been observed in the striatum (see e.g. Berke et al., 2004; Courtemanche et al., 2003). In addition, Tsai et al., (2009) demonstrated the relationship of high frequency oscillatory activity in reward and learning using an optogenetic approach to control the dopaminergic VTA activity in rats. The animals performed a conditioned place preference task which exposed them to two equivalent locations that were differentially associated with either $1 \mathrm{~Hz}$ or $50 \mathrm{~Hz}$ optical stimulation of the dopaminergic VTA neurons. Trained rats showed a clear preference for the location associated with the $50 \mathrm{~Hz}$-stimulation suggesting a role for oscillations at this frequency in associative learning. Other studies have also shown an increase of beta (Sturman and Moghaddam, 2012) and gamma (Berke, 2009; van der Meer and Redish, 2009) oscillatory activities in Ventral and Dorsal Striatum after 
reward delivery. In a recent study in monkeys performing a movement, Feingold (2011) found that beta activity occurred at the offset of a movement rather than during the movement itself. Moreover, beta activity in the striatum and prefrontal cortex was higher for correct than for incorrect trials, and presented an increased coherence in these two areas. On the basis of these findings, the authors proposed that beta activity indexed post-action reinforcement of actions associated with desired outcomes.

Given all these results, we propose a model of $\beta \gamma$ activity increase after unexpected and / or highly relevant positive outcomes. In Figure 3 we represent the main components of the reward network and their anatomical connections, which are involved in learning and motivation (Camara et al., 2009). The critical areas of the hippocampus - NAcc - VTA loop (as proposed in Axmacher et al., 2010) are shown connected in blue, while the other major connections are depicted in black. Several components of this circuit have been associated with oscillatory activity involved in both the coordination of distant brain areas and the coordination of local activity. The latter usually involves a cross-frequency coupling mechanisms, in which two different frequency bands act synchronously to integrate current state (driven by low frequency activity) with local activity (spikes or burst of activity of a group of neurons, Cohen et al., 2009). Therefore, encoding of new information is processed in the hippocampus on the basis of theta-gamma coupling (Colgin, 2011; Nyhus and Curran, 2010). In contrast, reward processing is mediated in the NAcc through oscillatory coupling between phase of alpha band and gamma power increase (Cohen et al., 2009). In addition, some other brain rhythm(s) are involved in the coordination of distant areas of the circuit. According to Fujisawa and Buzsáki, (2011), theta oscillatory activity is responsible for the coordination of the activity of VTA, hippocampus and prefrontal cortex while coherence in the gamma band would mediate hippocampus and NAcc integration (Ma and Leung, 2010). Finally, the circuit is completed with the connectivity between prefrontal cortex and ventral striatum (Figure 3, red thick line). We propose that $\beta \gamma$ oscillatory activity might be the key oscillatory mechanism in the coupling of frontostriatal areas, and that the $\beta \gamma$ power increase observed after unexpected and / or highly relevant positive outcomes might be modulated by this coupling. This proposal is based on previous studies implicating $\beta \gamma$ activity in the functional coupling of distant brain regions (Bressler et al., 1993; Steriade, 2006). Importantly, computational models (Kopell et al., 2000), receiving support from experimental animal data (Brovelli et al., 
2004), have shown that oscillations in the beta range $(<30 \mathrm{~Hz})$ are able to synchronize distant brain areas, in contrast to gamma oscillations $(30-80 \mathrm{~Hz})$ which would be used at local level. This would suggest that the high beta-low gamma range $(20-35 \mathrm{~Hz})$ found in EEG and MEG studies is an ideal mechanism for the coupling of frontostriatal structures. Indeed, the activity found in these studies is likely to reflect the local activity of groups of neurons located in the anterior Cingulate Cortex or ventromedial Prefrontal Cortex (Figure 3), but should be influenced by the activity of the other components of the reward system. We further hypothesize that $\beta \gamma$ activity transmits a fast motivational value signal to reward downstream brain structures through the fronto-striatal anatomical connection shown in the thick red line in Figure 3 connecting frontal cortex and NAcc. This tags those actions or stimuli that have produced better than expected results which then would be stored in memory. Importantly, prefrontal areas receive direct inputs from the medial posterior part of the Ventral Tegmental Area (Figure 3). These VTA neurons projecting to mPFC (as well as those projecting to NAcc core and medial shell) fire at frequencies between $20-30 \mathrm{~Hz}$ frequencies, in contrast to VTA dopaminergic neurons projecting to dorsolateral striatum and NAcc lateral shell which fire at frequencies below $10 \mathrm{~Hz}$ (Lammel et al., 2008; Walsh and Han, 2014). Therefore, VTA projections might modulate the activity of neurons in $\mathrm{mPFC}$ which in turn generate the $\beta \gamma$ power increase after unexpected gains or losses.

\section{Future Directions and Conclusions}

In the present review we have proposed that the $\beta \gamma$ activity is used to synchronize the different regions of the reward network after unexpected or relevant positive outcomes. However, more studies are needed in order to confirm this proposal. First, most of the previous studies have been performed using measures of power, and very little is known about other oscillatory characteristics of this component, such as phase coherence or inter-site-phase-clustering (Cohen et al., 2014). It has been proposed that phase synchrony might be a crucial mechanism in the communication among distant structures. Therefore, it would be important to study other oscillatory measures in the $\beta \gamma$ range in order to determine to which extent the activity observed might also present changes in phase and in the synchronization of distant sites/areas. In addition, most of the studies showing $\beta \gamma$ increase have been performed using EEG and MEG. In 
spite of the limited spatial resolution of these techniques, source analysis models of power data could identify the generators of the frontocentral $\beta \gamma$ activity. Complementary EEG-fMRI studies using mathematical approaches to combine the information of these two techniques (such as, e.g., Joint ICA, Calhoun et al., 2006, Doñamayor et al., 2012) could determine whether the activation found in M/EEG might be associated to activation in subcortical reward areas. Finally, intracranial recordings after the implantation of deep brain stimulation (DBS) electrodes for the treatment of certain neurologic and psychiatric diseases in combination with surface recordings could shed further light on $\beta \gamma$ activity (Münte et al., 2008; Herrojo-Ruiz et al., 2014). Indeed, target structures for DBS include the NAcc (e.g., in Obsessive Compulsive Disease, depression and alcoholism) as well as the globus pallidus internus and the subthalamic nucleus. The application of gambling paradigms, similar to those used in EEG and MEG studies, in this DBS studies might help in determining the role of the $\beta \gamma$ component in reward processing.

The present proposal might also be useful in the study of affective disorders and addictions. Previous studies have revealed that these disorders present abnormal activation of regions of the reward network after rewarding events and are characterized by an abnormal interaction between the Ventral Striatum and the prefrontal cortex (Pujada and Koenigs, 2014). Therefore, the study of $\beta \gamma$ oscillatory activity might help in determining how this impairment is affecting learning and attention, which at turn might help understanding other cognitive deficits associated to these pathologies.

Finally, it might be very important to study the role of $\beta \gamma$ oscillatory signatures in unexpected positive reward processing in other animal models using invasive electrical recordings and similar adapted paradigms. This type of research could be crucial for the discovery of the neural mechanisms involved in reward processing and the exact role of $\beta \gamma$ oscillatory activity in orchestrating these processes outlined in the present review. However, it is important to consider that generalization of the findings across species is not straightforward and in some cases no convergence might exists regarding neural oscillatory mechanisms (see for example, Narayanan et al., 2013). 


\section{Acknowledgements}

This project has been supported by Spanish grants from the MINECO (PSI2011-29219 to A.R.F and PSI2012-37472 to J.M.P.) and the Catalan Government (Generalitat de Catalunya, 2009 SGR 93). TFM is funded by the DFG and BMBF. 


\section{References}

Alexander, W.H., Brown, J.W., 2011. Medial prefrontal cortex as an action-outcome predictor. Nat. Neurosci. 14, 1338-44. doi:10.1038/nn.2921

Anderson, A.K., 2005. Affective influences on the attentional dynamics supporting awareness. J. Exp. Psychol. Gen. 134, 258-81. doi:10.1037/0096-3445.134.2.258

Axmacher, N., Cohen, M.X., Fell, J., Haupt, S., Dümpelmann, M., Elger, C.E., Schlaepfer, T.E., Lenartz, D., Sturm, V., Ranganath, C., 2010. Intracranial EEG correlates of expectancy and memory formation in the human hippocampus and nucleus accumbens. Neuron 65, 541-9. doi:10.1016/j.neuron.2010.02.006

Berke, J.D., 2009. Fast oscillations in cortical-striatal networks switch frequency following rewarding events and stimulant drugs. Eur.J.Neurosci. 30, 848-859. doi:10.1111/j.1460-9568.2009.06843.x

Berke, J.D., Okatan, M., Skurski, J., Eichenbaum, H.B., 2004. Oscillatory entrainment of striatal neurons in freely moving rats. Neuron 43, 883-96. doi:10.1016/j.neuron.2004.08.035

Berlyne, D.E., 1960. Conflict, arousal, and curiosity. McGraw-Hill series in psychology. McGraw-Hill, New York.

Berridge, K.C., Robinson, T.E., 1998. What is the role of dopamine in reward: hedonic impact, reward learning, or incentive salience? Brain Res. Brain Res. Rev. 28, 309-69.

Bilder, R.M., Volavka, J., Lachman, H.M., Grace, A.A., 2004. The catechol-Omethyltransferase polymorphism: relations to the tonic-phasic dopamine hypothesis and neuropsychiatric phenotypes. Neuropsychopharmacology 29, 1943-1961.

Blanchard, M.M., Mendelsohn, D., Stamp, J.A., 2009. The HR/LR model: Further evidence as an animal model of sensation seeking. Neurosci. Biobehav. Rev. 33, 1145-54. doi:10.1016/j.neubiorev.2009.05.009

Bressler, S.L., Coppola, R., Nakamura, R., 1993. Episodic multiregional cortical coherence at multiple frequencies during visual task performance. Nature 366, $153-156$.

Brovelli, A., Ding, M., Ledberg, A., Chen, Y., Nakamura, R., Bressler, S.L., 2004. Beta oscillations in a large-scale sensorimotor cortical network: directional influences revealed by Granger causality. Proc. Natl. Acad. Sci. U. S. A. 101, 9849-54. doi:10.1073/pnas.0308538101

Brown, P., Oliviero, A., Mazzone, P., Insola, A., Tonali, P., Di Lazzaro, V., 2001. Dopamine dependency of oscillations between subthalamic nucleus and pallidum in Parkinson's disease. J. Neurosci. 21, 1033-8. 
Bunzeck, N., Guitart-Masip, M., Dolan, R.J., Düzel, E., 2011. Contextual novelty modulates the neural dynamics of reward anticipation. J. Neurosci. 31, 12816-22. doi:10.1523/JNEUROSCI.0461-11.2011

Buschman, T.J., Miller, E.K., 2009. Serial, covert shifts of attention during visual search are reflected by the frontal eye fields and correlated with population oscillations. Neuron 63, 386-96. doi:10.1016/j.neuron.2009.06.020

Buzsaki, G., Draguhn, A., 2004. Neuronal oscillations in cortical networks. Science (80. ). 304, 1926-1929.

Camara, E., Rodriguez-Fornells, A., Munte, T.F., 2010. Microstructural Brain Differences Predict Functional Hemodynamic Responses in a Reward Processing Task. J. Neurosci. 30, 11398-11402. doi:10.1523/JNEUROSCI.0111-10.2010

Camara, E., Rodriguez-Fornells, A., Ye, Z., Munte, T.F., 2009. Reward networks in the brain as captured by connectivity measures. Front. Neurosci.

Cavanagh, J.F., Figueroa, C.M., Cohen, M.X., Frank, M.J., 2012. Frontal theta reflects uncertainty and unexpectedness during exploration and exploitation. Cereb. Cortex 22, 2575-86. doi:10.1093/cercor/bhr332

Chen, J., Lipska, B.K., Halim, N., Ma, Q.D., Matsumoto, M., Melhem, S., Kolachana, B.S., Hyde, T.M., Herman, M.M., Apud, J., Egan, M.F., Kleinman, J.E., Weinberger, D.R., 2004. Functional analysis of genetic variation in catechol-Omethyltransferase (COMT): effects on mRNA, protein, and enzyme activity in postmortem human brain. Am.J.Hum.Genet. 75, 807-821.

Cohen, M.X., Axmacher, N., Lenartz, D., Elger, C.E., Sturm, V., Schlaepfer, T.E., 2009. Good vibrations: cross-frequency coupling in the human nucleus accumbens during reward processing. J. Cogn. Neurosci. 21, 875-89. doi:10.1162/jocn.2009.21062

Cohen, M.X., Elger, C.E., Ranganath, C., 2007. Reward expectation modulates feedback-related negativity and EEG spectra. Neuroimage 35, 968-978.

Cohen, M.X., Ranganath, C., 2007. Reinforcement learning signals predict future decisions. J.Neurosci. 27, 371-378.

Cohen, M.X., Wilmes, K., Vijver, I. van de, 2011. Cortical electrophysiological network dynamics of feedback learning. Trends Cogn. Sci. 15, 558-66. doi:10.1016/j.tics.2011.10.004

Colgin, L.L., 2011. Oscillations and hippocampal-prefrontal synchrony. Curr. Opin. Neurobiol. 21, 467-74. doi:10.1016/j.conb.2011.04.006

Courtemanche, R., Fujii, N., Graybiel, A.M., 2003. Synchronous, focally modulated beta-band oscillations characterize local field potential activity in the striatum of awake behaving monkeys. J.Neurosci. 23, 11741-11752. 
Cunillera, T., Fuentemilla, L., Periañez, J., Marco-Pallarès, J., Krämer, U.M., Càmara, E., Münte, T.F., Rodríguez-Fornells, A., 2012. Brain oscillatory activity associated with task switching and feedback processing. Cogn. Affect. Behav. Neurosci. 12, 16-33. doi:10.3758/s13415-011-0075-5

Doñamayor, N., Marco-Pallarés, J., Heldmann, M., Schoenfeld, M.A., Münte, T.F., 2011. Temporal dynamics of reward processing revealed by magnetoencephalography. Hum. Brain Mapp. 32, 2228-40. doi:10.1002/hbm.21184

Doñamayor, N., Schoenfeld, M.A., Münte, T.F., 2012. Magneto- and electroencephalographic manifestations of reward anticipation and delivery. Neuroimage 62, 17-29. doi:10.1016/j.neuroimage.2012.04.038

Donchin, E., 1981. Surprise!? Surprise? Psychophysiology 18, 493-513. doi:10.1111/j.1469-8986.1981.tb01815.x

Engel, A.K., Fries, P., 2010. Beta-band oscillations--signalling the status quo? Curr. Opin. Neurobiol. 20, 156-65. doi:10.1016/j.conb.2010.02.015

Feingold, J., 2011. Beta Oscillations in Frontal Cortex and Striatum Represent PostProcessing of Successful Behavior. Massachusetts Institute of Technology.

Fiorillo, C.D., Tobler, P.N., Schultz, W., 2003. Discrete coding of reward probability and uncertainty by dopamine neurons. Science 299, 1898-1902.

Fuentemilla, L., Cucurell, D., Marco-Pallarés, J., Guitart-Masip, M., Morís, J., Rodríguez-Fornells, A., 2013. Electrophysiological correlates of anticipating improbable but desired events. Neuroimage 78, 135-44. doi:10.1016/j.neuroimage.2013.03.062

Fujisawa, S., Buzsáki, G., 2011. A 4 Hz oscillation adaptively synchronizes prefrontal, VTA, and hippocampal activities. Neuron 72, 153-65. doi:10.1016/j.neuron.2011.08.018

Gregoriou, G.G., Gotts, S.J., Zhou, H., Desimone, R., 2009. High-frequency, long-range coupling between prefrontal and visual cortex during attention. Science 324, $1207-$ 10. doi:10.1126/science. 1171402

HajiHosseini, A., Rodríguez-Fornells, A., Marco-Pallarés, J., 2012. The role of betagamma oscillations in unexpected rewards processing. Neuroimage 60, 1678-85. doi:10.1016/j.neuroimage.2012.01.125

Herrmann, C.S., Knight, R.T., 2001. Mechanisms of human attention: event-related potentials and oscillations. Neurosci. Biobehav. Rev. 25, 465-76.

Herrojo Ruiz, M., Huebl, J., Schönecker, T., Kupsch, A., Yarrow, K., Krauss, J.K., Schneider, G.-H., Kühn, A.A., 2014. Involvement of human internal globus 
pallidus in the early modulation of cortical error-related activity. Cereb. Cortex 24, 1502-17. doi:10.1093/cercor/bht002

Jensen, O., Kaiser, J., Lachaux, J.-P., 2007. Human gamma-frequency oscillations associated with attention and memory. Trends Neurosci. 30, 317-24. doi:10.1016/j.tins.2007.05.001

Kawasaki, M., Yamaguchi, Y., 2013. Frontal theta and beta synchronizations for monetary reward increase visual working memory capacity. Soc. Cogn. Affect. Neurosci. 8, 523-30. doi:10.1093/scan/nss027

Keil, A., Müller, M.M., Gruber, T., Wienbruch, C., Elbert, T., 2001. Human large-scale oscillatory brain activity during an operant shaping procedure. Brain Res. Cogn. Brain Res. 12, 397-407.

Knutson, B., Taylor, J., Kaufman, M., Peterson, R., Glover, G., 2005. Distributed neural representation of expected value. J.Neurosci. 25, 4806-4812.

Kopell, N., Ermentrout, G.B., Whittington, M.A., Traub, R.D., 2000. Gamma rhythms and beta rhythms have different synchronization properties. Proc. Natl. Acad. Sci. U. S. A. 97, 1867-72.

Lalo, E., Thobois, S., Sharott, A., Polo, G., Mertens, P., Pogosyan, A., Brown, P., 2008. Patterns of bidirectional communication between cortex and basal ganglia during movement in patients with Parkinson disease. J. Neurosci. 28, 3008-16. doi:10.1523/JNEUROSCI.5295-07.2008

Lammel, S., Hetzel, A., Häckel, O., Jones, I., Liss, B., Roeper, J., 2008. Unique properties of mesoprefrontal neurons within a dual mesocorticolimbic dopamine system. Neuron 57, 760-73. doi:10.1016/j.neuron.2008.01.022

Lapray, D., Bergeler, J., Luhmann, H.J., 2009. Stimulus-induced gamma activity in the electrocorticogram of freely moving rats: the neuronal signature of novelty detection. Behav. Brain Res. 199, 350-4. doi:10.1016/j.bbr.2008.12.028

Legault, M., Wise, R.A., 2001. Novelty-evoked elevations of nucleus accumbens dopamine: dependence on impulse flow from the ventral subiculum and glutamatergic neurotransmission in the ventral tegmental area. Eur. J. Neurosci. 13, 819-28.

Leicht, G., Troschütz, S., Andreou, C., Karamatskos, E., Ertl, M., Naber, D., Mulert, C., 2013. Relationship between Oscillatory Neuronal Activity during Reward Processing and Trait Impulsivity and Sensation Seeking. PLoS One 8, e83414. doi:10.1371/journal.pone.0083414

Lisman, J.E., Grace, A.A., 2005. The hippocampal-VTA loop: controlling the entry of information into long-term memory. Neuron 46, 703-13. doi:10.1016/j.neuron.2005.05.002 
Llinás, R., Urbano, F.J., Leznik, E., Ramírez, R.R., van Marle, H.J.F., 2005. Rhythmic and dysrhythmic thalamocortical dynamics: GABA systems and the edge effect. Trends Neurosci. 28, 325-33. doi:10.1016/j.tins.2005.04.006

Ma, J., Leung, L.S., 2010. Kindled seizure in the prefrontal cortex activated behavioral hyperactivity and increase in accumbens gamma oscillations through the hippocampus. Behav. Brain Res. 206, 68-77. doi:10.1016/j.bbr.2009.08.038

Marco-Pallares, J., Cucurell, D., Cunillera, T., García, R., Andrés-Pueyo, A., Munte, T.F., Rodriguez-Fornells, A., 2008. Human Oscillatory Activity Associated to Reward Processing in a Gambling Task. Neuropsychologia 46, 241-248.

Marco-Pallarés, J., Cucurell, D., Cunillera, T., Krämer, U.M., Càmara, E., Nager, W., Bauer, P., Schüle, R., Schöls, L., Münte, T.F., Rodriguez-Fornells, A., 2009. Genetic variability in the dopamine system (dopamine receptor D4, catechol-Omethyltransferase) modulates neurophysiological responses to gains and losses. Biol. Psychiatry 66, 154-61. doi:10.1016/j.biopsych.2009.01.006

Mas-Herrero, E., Marco-Pallarés, J., 2014. Frontal Theta Oscillatory Activity Is a Common Mechanism for the Computation of Unexpected Outcomes and Learning Rate. J. Cogn. Neurosci. 26, 447-458. doi:10.1162/jocn_a_00516

Matsumoto, M., Hikosaka, O., 2009. Two types of dopamine neuron distinctly convey positive and negative motivational signals. Nature 459, 837-841.

Meehl, P.E., 1975. Hedonic capacity: some conjectures. Bull. Menninger Clin. 39, 295307.

Mellers, B., McGraw, A., 2001. Anticipated emotions as guides to choice. Curr. Dir. Psychol. 10, 201-214

Mellers, B.A., Schwartz, A., Cooke, A.D., 1998. Judgment and decision making. Annu. Rev. Psychol. 49, 447-77. doi:10.1146/annurev.psych.49.1.447

Narayanan, N.S., Cavanagh, J.F., Frank, M.J., Laubach, M., 2013. Common medial frontal mechanisms of adaptive control in humans and rodents. Nat. Neurosci. 16, 1888-95. doi:10.1038/nn.3549

Nieuwenhuis, S., Slagter, H.A., von Geusau, N.J.A., Heslenfeld, D.J., Holroyd, C.B., 2005. Knowing good from bad: differential activation of human cortical areas by positive and negative outcomes. Eur. J. Neurosci. 21, 3161-3168.

Nyhus, E., Curran, T., 2010. Functional role of gamma and theta oscillations in episodic memory. Neurosci. Biobehav. Rev. 34, 1023-35. doi:10.1016/j.neubiorev.2009.12.014

O’Doherty, J., Rolls, E.T., Francis, S., Bowtell, R., McGlone, F., 2001. Representation of pleasant and aversive taste in the human brain. J. Neurophysiol. 85, 1315-21. 
Ohman, A., Flykt, A., Esteves, F., 2001. Emotion drives attention: detecting the snake in the grass. J. Exp. Psychol. Gen. 130, 466-78.

Olson, J.M., Roese, N.J., Zanna, M.P., 1996. Expectancies, in: Higgins, E.T., Kruglanski, A.W. (Eds.), Social Psychology: Handbook of Basic Principles. Guilford, New York, pp. 211-238.

Ortony, A., Glore, G.L., Collins, A., 1998. The cognitive structure of emotions. Cambridge University Press

Padrão, G., Mallorquí, A., Cucurell, D., Marco-Pallares, J., Rodriguez-Fornells, A., 2013. Neurophysiological differences in reward processing in anhedonics. Cogn. Affect. Behav. Neurosci. 13, 102-15. doi:10.3758/s13415-012-0119-5

Pessiglione, M., Schmidt, L., Draganski, B., Kalisch, R., Lau, H., Dolan, R.J., Frith, C.D., 2007. How the brain translates money into force: a neuroimaging study of subliminal motivation. Science 316, 904-6. doi:10.1126/science.1140459

Pessoa, L., Kastner, S., Ungerleider, L.G., 2002. Attentional control of the processing of neural and emotional stimuli. Brain Res. Cogn. Brain Res. 15, 31-45.

Phaf, R.H., Rotteveel, M., 2012. Affective monitoring: a generic mechanism for affect elicitation. Front. Psychol. 3, 47. doi:10.3389/fpsyg.2012.00047

Ribary, U., 2005. Dynamics of thalamo-cortical network oscillations and human perception. Prog. Brain Res. 150, 127-42. doi:10.1016/S0079-6123(05)50010-4

Russo, S.J., Nestler, E.J., 2013. The brain reward circuitry in mood disorders. Nat. Rev. Neurosci. 14, 609-25. doi:10.1038/nrn3381

Schachter, S., Singer, J., 1962. Cognitive, social, and physiological determinants of emotional state. Psychol. Rev. 69, 379-399

Schnitzler, A., Gross, J., 2005. Normal and pathological oscillatory communication in the brain. Nat. Rev. Neurosci. 6, 285-96. doi:10.1038/nrn1650

Schultz, W., Apicella, P., Ljungberg, T., 1993. Responses of monkey dopamine neurons to reward and conditioned stimuli during successive steps of learning a delayed response task. J.Neurosci. 13, 900-913.

Schultz, W., Dayan, P., Montague, P.R., 1997. A neural substrate of prediction and reward. Science 275, 1593-1599.

Sokolov, E.N., 1963. Higher nervous functions; the orienting reflex. Annu. Rev. Physiol. 25, 545-80. doi:10.1146/annurev.ph.25.030163.002553

Steriade, M., 2006. Grouping of brain rhythms in corticothalamic systems. Neuroscience 137, 1087-106. doi:10.1016/j.neuroscience.2005.10.029 
Sturman, D.A., Moghaddam, B., 2012. Striatum processes reward differently in adolescents versus adults. Proc. Natl. Acad. Sci. U. S. A. 109, 1719-24. doi:10.1073/pnas.1114137109

Tobler, P.N., Fiorillo, C.D., Schultz, W., 2005. Adaptive coding of reward value by dopamine neurons. Science (80-. ). 307, 1642-1645.

Tsai, H.-C., Zhang, F., Adamantidis, A., Stuber, G.D., Bonci, A., de Lecea, L., Deisseroth, K., 2009. Phasic firing in dopaminergic neurons is sufficient for behavioral conditioning. Science 324, 1080-4. doi:10.1126/science.1168878

Uhlhaas, P.J., Singer, W., 2006. Neural synchrony in brain disorders: relevance for cognitive dysfunctions and pathophysiology. Neuron 52, 155-68. doi:10.1016/j.neuron.2006.09.020

Van de Vijver, I., Ridderinkhof, K.R., Cohen, M.X., 2011. Frontal oscillatory dynamics predict feedback learning and action adjustment. J. Cogn. Neurosci. 23, 4106-21. doi:10.1162/jocn_a_00110

Van der Meer, M.A.A., Redish, A.D., 2009. Low and High Gamma Oscillations in Rat Ventral Striatum have Distinct Relationships to Behavior, Reward, and Spiking Activity on a Learned Spatial Decision Task. Front. Integr. Neurosci. 3, 9. doi:10.3389/neuro.07.009.2009

Walsh, J.J., Han, M.H., 2014. The heterogeneity of ventral tegmental area neurons: Projection functions in a mood-related context. Neuroscience 282C, 101-108. doi:10.1016/j.neuroscience.2014.06.006

Wang, X.-J., 2010. Neurophysiological and computational principles of cortical rhythms in cognition. Physiol. Rev. 90, 1195-268. doi:10.1152/physrev.00035.2008

Womelsdorf, T., Fries, P., 2007. The role of neuronal synchronization in selective attention. Curr. Opin. Neurobiol. 17, 154-60. doi:10.1016/j.conb.2007.02.002

Zillmann, D., 1978. Attribution and misattribution of excitatory reactions, in: Harvey, J.H., Ickes, W.J., Kidd, R.F. (Eds.), New Directions in Attribution Research. Lawrence Erlaub Associates, Hillsdale, NJ, pp. 335-368. 


\section{Figure}

A

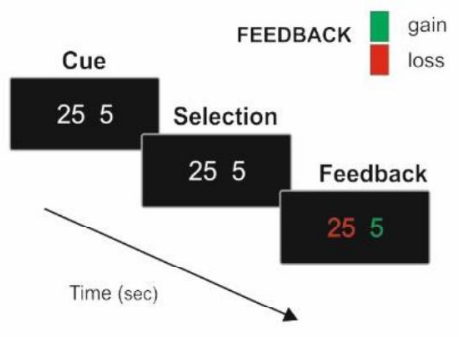

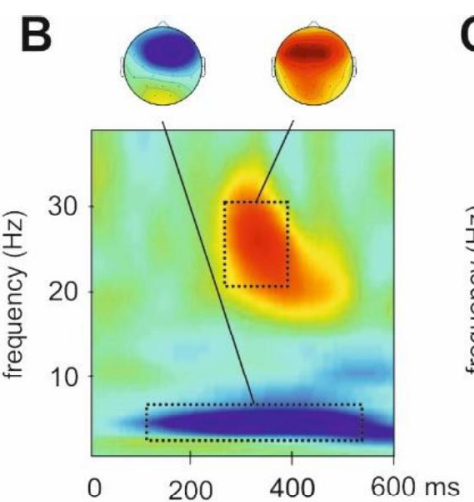

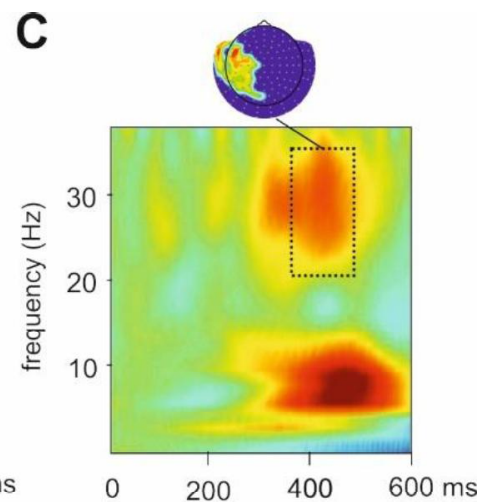

Figure 1. $\beta \gamma$ oscillatory activity after monetary gains in gambling paradigms. A. Monetary gambling paradigm used to describe the oscillatory components of feedback processing in Marco-Pallarés et al., 2008. In each trial, the participant had to bet on one of the two numbers presented. If the number turned green, the participant won the indicated amount of money (in Euro cent); if turned red, s/he lost it. B. Time frequency decomposition of the EEG oscillatory activity recorded from the scalp for the gain minus loss conditions. Note the increase in theta activity for losses (in blue) and the increase of $\beta \gamma$ activity for gains (red) 200-400 ms after feedback delivery (scalp distribution in the top; adapted from Marco-Pallares et al., 2008) C. Time frequency MEG activity of the gain minus loss conditions using the same paradigm. A similar $\beta \gamma$ activity was found 300-500 ms after reward presentation (adapted from Doñamayor et al., 2011). Note the difference in the topographies in $\mathrm{B}$ and $\mathrm{C}$ due to the different techniques used in the two experiments (EEG in B and MEG in C). 

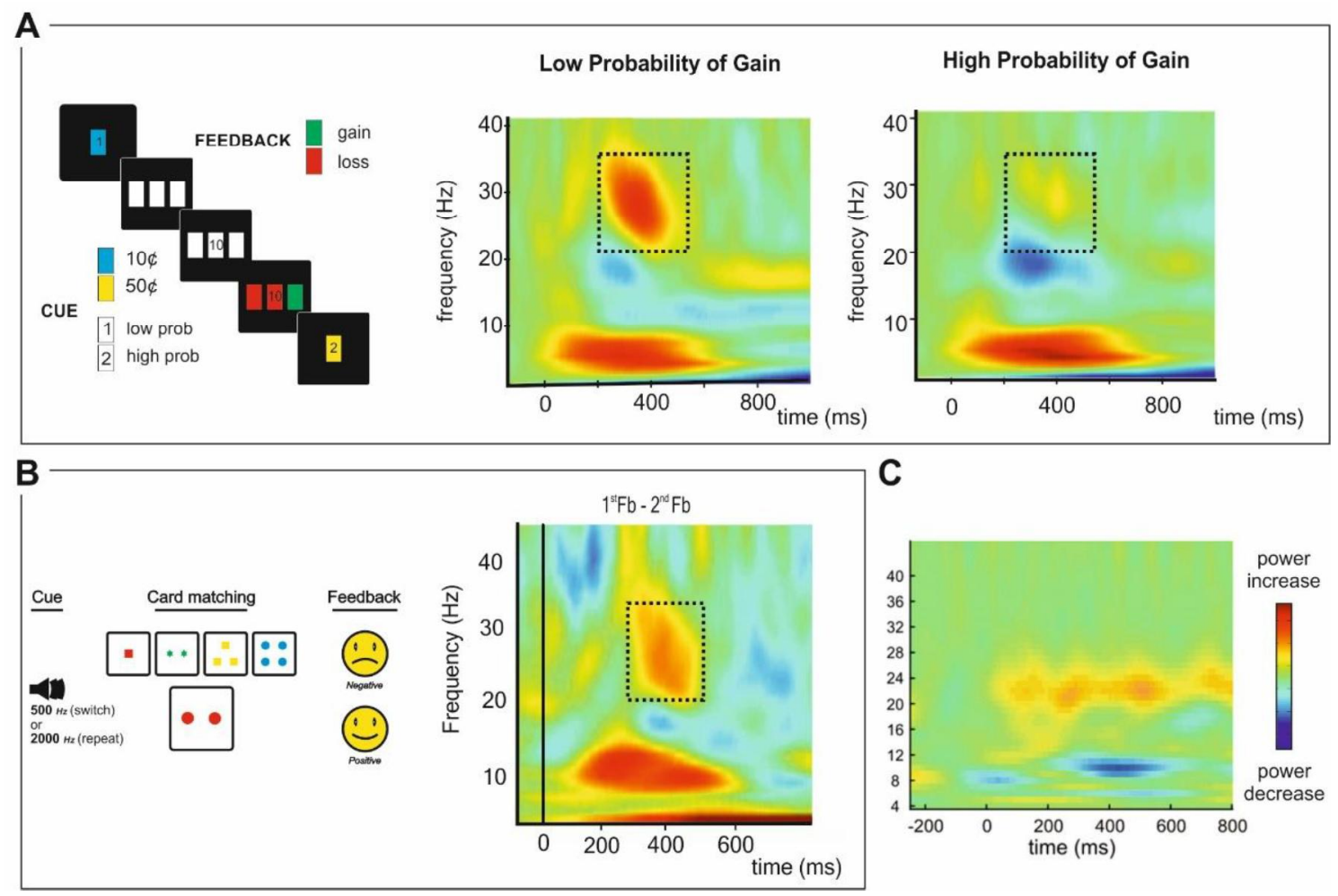

Figure 2. $\beta \gamma$ oscillatory activity in relevant positive feedbacks and reward expectation. A. Time frequency data for gain conditions in a feedback-guided decision tasks in Hajihosseini et al., 2012. Left panel indicates the experimental paradigm used in the experiment. In each trial, a cue indicated the magnitude (color of the card) and the probability (number of the card) of the future reward and punishment. Then participants had to select between one of the three cards displayed on the screen and then feedback indicated whether participant won or lost certain amount of money. Wavelet analysis showed the oscillatory activity for gain condition in trials with low probability of winning (medium panel) and high probability of winning (right panel). Note the increase in $\beta \gamma$ activity in the low probability of winning condition (adapted from HajiHosseini et al., 2012). B. Left. Wisconsin Sorting Card Task paradigm used in Cunillera et al., (2012). Participants had to respond following a rule. Feedback indicated the correctness of the response given. After some trials, the rule changed. The timefrequency representation shows the difference between the first and second positive feedbacks indicating a correct response after a rule change. Note the enhancement in the beta band for the first positive feedback which provides the most relevant and novel information compared to the second one (adapted from Cunillera et al., 2012). C. MEG beta increase of activity after a cue predicting high probability of obtaining reward (adapted from Bunzeck et al., 2011). In a previous phase of the experiment, some cues 
were associated to different probabilities of reward $(0,0.4$ and 0.8$)$. In a latter phase, some novel or familiar images were presented and participants were instructed to respond as fast as possible. After the response, the cues associated to different probabilities appeared. As shown in the image, the anticipation of a probable reward elicited a beta power increase which was more pronounced in those conditions implying novelty. 


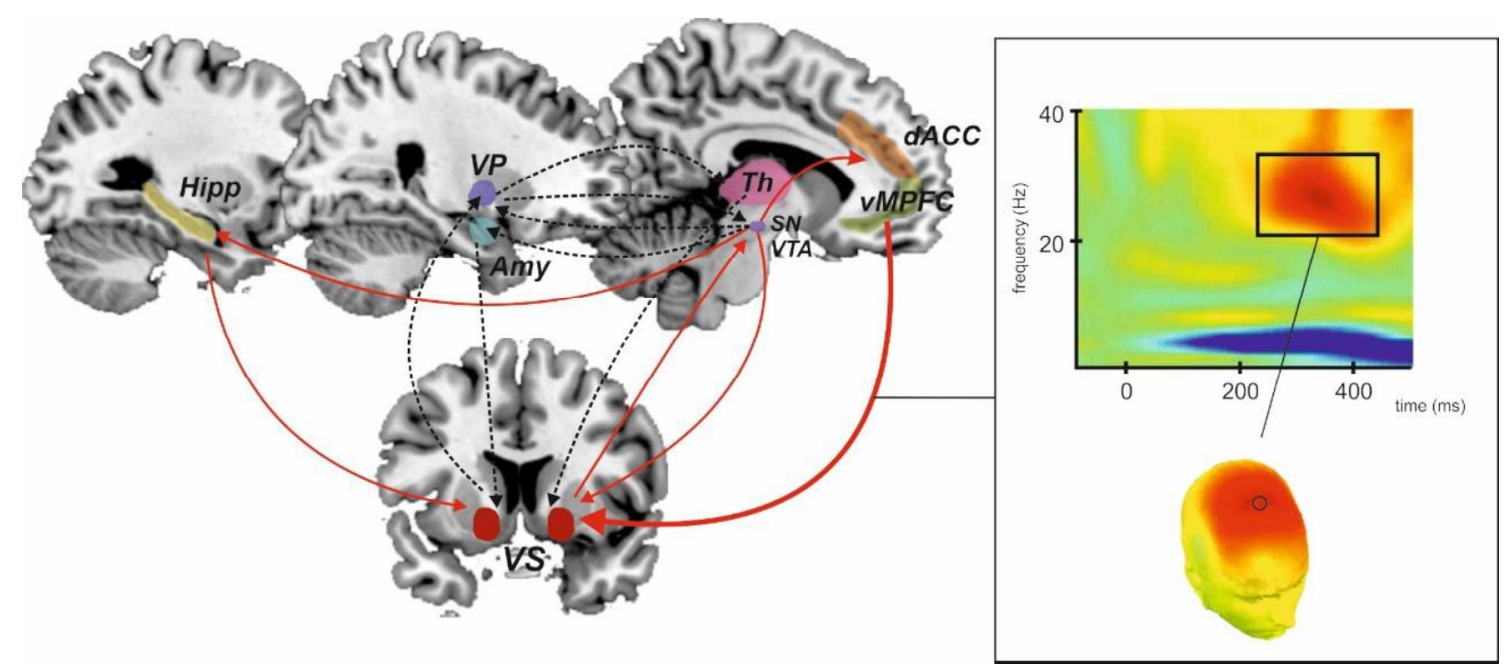

Figure 3. Model linking $\beta \gamma$ activity to unexpected or highly relevant positive outcomes. Arrows indicate the anatomical connections of the different components of the reward system. In red, the components of the hippocampus - NAcc - VTA loop proposed in Axmacher et al., (2010). The $\beta \gamma$ oscillatory activity is generated in the prefrontal cortex, probably at the $\mathrm{AACC}$ or vMPFC and is influenced by the VTA activity (red arrow). Finally, in our model we propose that the $\beta \gamma$ activity is an ideal mechanism for the coupling of frontostriatal structures to transmit a fast motivational value signal downstream to the reward network (red thick line).

dACC: dorsal Anterior Cingulate Cortex; vMPFC: ventromedial Prefrontal Cortex; Th: Thalamus; SN/VTA: Sustantia Nigra/ Ventral Tegmental Area; VP: Ventral Pallidum; Amy: Amygdala; Hipp: Hippocampus; VS: Ventral Striatum. 